\title{
Peripherally inserted central catheter complications in neonates - our experiences
}

\author{
STOJISLAV KONJEVIĆ, DARIO DJUKIĆ, BARBARA STANIMIROVIĆ, ANA BLAGOJEVIĆ, VANJA BOBIĆ, BILJANA BANJA \\ Paediatric Clinic, University Hospital Clinical Centre Banja Luka, Bosnia and Herzegovina \\ Corresponding author: \\ Stojislav Konjević \\ Pediatric Clinic \\ University Hospital, Clinical Centre \\ 12 beba bb, 78000 Banja Luka \\ Bosnia and Herzegovina \\ Phone: 0038751342405 \\ E-mail: stojislav.konjevic@gmail.com
}

\section{ABSTRACT}

The aim of this study was to investigate the incidence and risk factors of peripherally inserted central venous catheters (PICCs) in critically ill premature neonates; A retrospective analysis of 30 infants with very low and extremely low birth weights (VLBW, ELBW) who underwent PICC placement over a three-year period, from January 2012 till January 2015. Gestational age, birth weight (BW), sex, site of catheter placement, reason for catheter removal, duration of catheter use, proven sepsis, type of reported organism and rate of complications were collected. The infants were classified into two groups according to BWs: Group 1-VLBW infants (BW between 1,000 and 1,500 g) and Group 2ELBW infants (BW $<1,000 \mathrm{~g}$ ). During the study period PICCs were attempted in 40 patients. A PICC was successfully inserted into 30 patients $(75 \%)$. PICCs placed in either the upper or the lower extremity. There were no differences in complication rates. The median time of catheter insertion was 13 (1-35) days for Group 1 and 11 (6-19) days for Group 2. The median duration of PICCs was 10.5 (2-16) and 12.2 (3-25) days. Statistical analysis showed that there was a significant difference between the groups for both catheter insertion day and mean duration of PICCs $(\mathrm{p}=0.241$, respectively). There were no significant differences between groups for the reasons for catheter removal ( $\mathrm{p}=0.598)$. PICCs are convenient for the administration of long course antibiotics and parenteral nutrition for both VLBW and ELBW infants, but there are many risks associated with the insertion of PICCs, including serious and fatal complications.
Key words: Catheter-related infections, Central venous catheterization, Complications, Extremely low birth weight infant, Very low birth weight infant

\section{INTRODUCTION}

Since their early descriptions over two decades ago, percutaneously inserted central catheters (PICCs) have become a mainstay in neonatal intensive care units for longterm venous access to deliver parenteral nutrition and medications. $(1,2)$ Peripherally inserted central venous catheters (PICCs) are widely used and are valuable in the management of VLBW and ELBW infants. PICCs provide an important route to deliver TPN, hyperosmolar solutions, cardiac inotropic agents and some medications or solutions that are considered as chemical irritants, especially when these premature infants are experiencing temporarily inadequate gastrointestinal tractintake. Also, decrease in stress and suffering associated with the handling of the infant with multiple peripheral intravenous lines start again. (3) Approximately 8.3-33\% of neonates admitted to an intensive care unit require PICC insertion. (4) The presence of an indwelling PICC also has relevant risks and morbidity, including vascular compromise, pleural and pericardial effusion, cardiac tamponade, obstruction, thrombosis, catheter-related sepsis (CRS), leakage, catheter migration, vegetations and phlebitis. (5)

\section{MATERIALS AND METHODS}

This study was conducted in a tertiary-level NICU at the Children's Hospital, Banja Luka, Bosnia and Hercegovina. Neonates with less than 1,500 g body weight and with a PICC for maintenance of intravenous fluid, antibiotics or TPN were eligible for enrolment. Patients were classified into two groups according to their BWs: Group 1 consisted of VLBW infants with BWs between 1,000 and $1500 \mathrm{~g}$, and Group 2 consisted of VLBW neonates with a BW $<1,000$ g (ELBW group).

\section{PICC placement and management}

A percutaneous central catheter (Nutriline Twinflow 2Fr, Vygon, Paris, France) was used in all patients. Fentanyl $(1-2 \mu \mathrm{g} / \mathrm{kg})$ was used for analgesia of the infant. Catheters were introduced aseptically, including the use of sterile gowns, caps and masks during the placement. Hand hygiene was performed with antiseptic-containing soap, and skin was disinfected with $70 \%$ alcohol before catheter insertion. All catheters were inserted by blind cannulation. Prior to insertion, the catheter length was measured according to the indwelling vein. The catheter was inserted via a pileable micoflush needle into an appropriate vein in the absence of any clinical sign of infection. The position of the catheters were confirmed radiografically. The catheters were secured with sterile adhesive strips and covered with sterile transparent adhesive dressing. Both the sterile adhesive strips and the transparent dressing were changed every seven days or when necessary. Data were collected retrospectively over a threeyear period. Informed consent was ob- 
tained from parents for PICC placement. The data included gestational age, BW, sex, site of catheter placement, reason for catheter removal, duration of catheter use, proven sepsis and type of the reported organism and rate of complications. Mortality during catheterization was also recorded. Catheter-related complications were categorized into three groups: mechanical, infectious and death. The withdrawal of the catheters were done when infants were tolerating full enteral feedings or if a prolonged intravenous medication course was completed. The PICCs were also removed if there were any signs of complications. The tips of the catheters were cultured routinely after the catheters were removed. If a bacterial growth was detected in the tip of a catheter without any clinical deterioration or without a positive blood culture, it was defined as bacterial colonization of the catheter. When clinical signs of sepsis were observed, a blood sample was drawn from a peripheral vein for blood culture. If a bacterial growth was detected in the blood culture, the PICC was removed and the tip of the catheter was cultured. CRS was defined as clinical deterioration and positive blood culture with the same microorganism present on the catheter tip and a clinical and microbiological absence of any other source of infection. The incidence rate of CRS was expressed as the number of infections per 1,000 catheter days.

Statistical analysis

Statistical Package for Social Sciences for Windows (SPSS version 15.0, SPSS Inc., Chicago, IL, USA) was used to process data and statistics. Data were expressed as mean \pm standard deviation (SD). The Mann-Whitney U-test was used to analyze the gestational age, BW, duration of PICCs patency and catheter insertion age. The Fischer exact test and Pearson Chi-square tests were used to analyze mechanical and infectious complications and death rate. $\mathrm{P}$ value $<0.05$ was considered to be statistically significant.

\section{RESULTS}

During the study period PICCs were attempted in 40 patients. A PICC was successfully inserted into 30 patients (75\%). There were no insertion-related complications in the study group. Only two infants required multiple PICCs for prolonged treatment. The insertion locations of the catheters were in the upper extremity in 12 PICCs (40\%), and in the lower extremity in 18 PICCs $(60 \%)$. There were 12 infants were in Group 1 and 18 in Group 2.

Descriptive data of the patients and the study are provided for in I. The median time of catheter insertion was 13 (1-35) days for Group 1 and 11 (6-19) days for Group 2. The median duration of PICCs was 10.5 (2-16) and 12.2 (3-25) days. Statistical analysis showed that there was a significant difference between the groups for both catheter insertion day and mean duration of PICCs ( $p=0.241$, respectively). The reasons for the removal of the catheters were: (a) completion of intravenous therapy in 10 cases (33.3\%), (b) occlusion in 11 cases $(36.6 \%)$, (c) leakage in 3 cases (10\%), (d) CRS in 5 cases (16.6\%), (e) phlebitis in 1 case (3.3\%) and (f) death in 2 cases $(6.6 \%)$. Bacterial colonization without systemic infection was found in 2 patients. CRS was found in 5 patients. Coagulasenegative Staphylococcus epidermidis was found in 3 patients, Candida albicans in 1 patient and Klebsiela pneumoniae spp in 1 patient. The incidence rate of CRS was found to be 13.47 per 1,000 catheter days. There were no statistically significant differences in the number of complication rates relating to the insertion location. Two infants died during the study. Both of them were in the ELBW group. One of them died due to intracranial haemorrhage, and the second infants died due to PICC complications (C. Albicans infection). There were significant differences between groups for the reasons of removal $(\mathrm{p}=0.598)$. The reasons for the removal of PICCs in relation to the groups are presented in table 2 .

\section{DISCUSSION}

PICCs have been increasingly used in premature infants in order to provide adequate parenteral nutrition, and administration of antibiotics and fluids. In the current study, the median duration in place of PICCs was 12.7 and 11 days for VLBW and ELBW infants, respectively.

Several studies have reported a significant number of complications due to PICCs. Some of them are major and potentially fatal, such as thrombosis, extravascular collection of fluid in the thorax, pericardial tamponade and perforation of the myocardium, pulmonary embolism, sepsis and death. (4 - 6) Mechanical complications of PICCs reported in previous studies includ- ed occlusion, accidental removal, leakage or breakage, catheter migration and phlebitis. (4 -7) The PICC-related mechanical complication rates are reported in a wide range from $0 \%$ to $48 \%$. $(1,2,4,7,8)$ As in other published studies, our study found that occlusion was the most common complication (36.6\%). Using the smallest PICC in the largest vein, adding heparin to the intravenous fluid and running fluids continuously are useful strategies for preventing occlusion. (9) Phlebitis was accepted in the presence of linear red streak developed along the superficial veins from the insertion site that occurred in 1 (5.5\%) patients. CRS is defined as clinical deterioration and a positive blood culture with the same microorganism present on the catheter tip and clinical and microbiological absence of any other source of infection. PICC-related sepsis rate ranged from $0 \%$ to $29 \%$ of placed catheters, resulting in 0.3 to 16.7 per 1,000 catheter days. (10) Suresh and Edwards reported CRS rates of 2.7 and 3.7 per 1,000 catheters days for VLBW and ELBW infants, respectively. $(11,12)$ Parellada et al and Cairns et al reported CRS rates of 8.21 per 1,000 catheter days, respectively. In both cases, the study populations had similar BWs and gestational ages as in our study. $(13,14)$ The CRS rate in our study was 13.47 per 1,000 catheter days. There was a significant difference in the VLBW and ELBW groups since the two deaths we had in our study were in the ELBW group. Our results confirm previous studies that coagulase-negative $\mathrm{S}$. epidermidis was the major organism associated with CRS. (5, $8,9,12$ ) Low BW, lesser gestational age, longer duration of PICC and femoral site insertion were previously reported to increase the risk of CRS. $(9,15,16)$ In our study, there were no statistically significant differences in the number of complication rates in relation to the insertion site. Also, the rate of complication of PICCs was not higher in infants with $\mathrm{BW}<1,000 \mathrm{~g}$ (ELBW group) than in infants with BW 1,000$1,500 \mathrm{~g}$ (VLBW group). The prevention of CRS is one of the priorities of most NICUs because of the potential for reductions in mortality, morbidity and hospitalization days.

There are some limitations to our study. The PICCs have very narrow diameters (1 or $2 \mathrm{Fr}$ ), and therefore these catheters could not be used for blood sampling or blood products transfusions. We could not collect a blood culture from the catheters. Therefore, the tips of the catheters 
were cultured from patients whose blood cultures were positive for bacterial growth. The incidence of failure at insertion and mechanical irreversible occlusion were high in our study. Ultrasound guidance and the use of near-infrared devices may reduce the rate of insertion failure, but we did not utilize these techniques. Heparin was not added routinely in maintenance fluids, TPN and lipid solutions but we flushed the catheters with heparin 10IU/ $\mathrm{ml}$ every six hours or as necessary.

\section{CONCLUSION}

This study confirmed that PICCs represent a suitable intravenous route for the administration of long course antibiotics and parenteral nutrition in premature neonates. Mechanical complications were the most common reason for removal. As in other published studies, our study found that occlusion was the most common complication (36.6\%). There were no statistically significant differences in the number of complication rates according to the insertion site. The ELBW group was increased risk of catheter complications in our study. It is important to emphasize the fact that CRS per 1000 catheter days was increased (13.47/1000 days) probably due to limited conditions. There are many risks associated with the insertion of PICCs including serious and fatal complications.

Table 1. Demographic characteristics of the groups

\begin{tabular}{llll}
\hline & $\begin{array}{l}\text { Group 1 (n=12) } \\
\text { Mean (min-max) }\end{array}$ & $\begin{array}{l}\text { Group 2 (n=18) } \\
\text { Mean (min-max) }\end{array}$ & p-Value \\
\hline Gestational age (wk) & $30.7(28-34)$ & $28.1(25-33)$ & 0.00634 \\
\hline Birth weight (g) & $1220.8(1050-1450)$ & $824.4(670-990)$ & 0.002 \\
\hline Male/female & $7 / 5$ & $11 / 7$ & 0.879072 \\
\hline Catheter insertion age (day) & $10.5(2-16)$ & $12.2(3-25)$ & 0.24196 \\
\hline Duration of catheter (day) & $12.7(5-30)$ & $11.0(3-30)$ & 0.19215 \\
\hline
\end{tabular}

Table 2. Reasons for removal of piccs

\begin{tabular}{llll}
\hline & $\begin{array}{l}\text { Group 1 (n=12) } \\
\text { Mean (min-max) }\end{array}$ & $\begin{array}{l}\text { Group 2 (n=18) } \\
\text { Mean (min-max) }\end{array}$ & p-Value \\
\hline No longer required & $5(41.6 \%)$ & $5(27.7 \%)$ & 0.429195 \\
\hline Occlusion & $4(33.3 \%)$ & $7(38.9 \%)$ & 0.75706 \\
\hline Leakage & $1(8.3 \%)$ & $2(11.1 \%)$ & 0.803785 \\
\hline Phlebitis & $0(0 \%)$ & $1(5.5 \%)$ & 0.406282 \\
\hline Catheter-related sepsis & $2(16.6 \%)$ & $3(16.6 \%)$ & 1 \\
\hline Catheter-related death & $0(0 \%)$ & $1(5.5 \%)$ & 0.406282 \\
\hline Overall mortality & $0(0 \%)$ & $2(11.1 \%)$ & 0.231998 \\
\hline
\end{tabular}

${ }^{\star}$ Overall mortality $=$ Catheter-related plus noncatheter-related death

\section{REFERENCES}

1. Shaw JL. Parenteral nutrition in the management of sick low birthweight infants. Pediatr Clin North Am 1973; $20: 333-58$.

2. Riordan TP. Placement of central venous lines in the premature infant. JPEN 1979; 3: 381-2.

3. Paulson PR, Miller KM. Neonatal peripherally inserted central catheters: recommendations for prevention of insertion and postinsertion complications. Neonatal Netw. 2008;27(4):245-257.

4. van den Hoogen A, Brouwer MJ, Gerards LJ, Fleer A, Krediet TG. Removal of percutaneously inserted central venous catheters innneonates is associated with the occurrence of sepsis. Acta Paediatr 2008;97:1250-2

5. Ohki Y, Yoshizawa Y, Watanabe M, Kuwashima M, Morikawa A. Complications of percutaneously inserted central venous catheters in Japanese neonates. Pediatr Int. 2008;50(5):636-639.

6. Darling JC, Newell SJ, Mohamdee O, Uzun O, Cullinane CJ, Dear PR. Central venous catheter tip in the right atrium: a risk factor for neonatal cardiac tamponade. J Perinatol. 2001;21(7):461-464.

7. Janes M, Kalyn A, Pinelli J, Hamilton BP. A randomized trial comparing peripherally inserted central venous catheters and peripheral intravenous catheters in infants with very low birth weight. J Pediatr Surg. 2000;35(7):1040-1044.

8. Bulbul A, Okan F, Nuhoglu A. Percutaneously inserted central catheters in the newborns: a center's experience in Turkey. J Matern Fetal Neonatal Med. 2010;23(6):529-535. 
9. Stolfi I, Boccanera F, Chiara C, et al. Central venous lines and how to manage them. Early Hum Dev. 2009;85(10Suppl):S83-S84

10. Borghesi A, Stronati M. Strategies for the prevention of hospital-acquired infections in the neonatal intensive care unit. J Hosp Infect. 2008;68(4):293-300.

11. Suresh GK, Edwards WH. Central line-associated bloodstream infections in neonatal intensive care: changing the mental model from inevitability to preventability. Am J Perinatol. 2012;29(1):57-64.

12. Klein JF, Shahrivar F. Use of percutaneous silastic central venous catheters in neonates and the management of infectiouscomplications. Am J Perinatol. 1992;9(4):261-264.

13. Parellada JA, Moïse AA, Hegemier S, Gest AL. Percutaneous central catheters and peripheral intravenous catheters have similar infection rates in very low birth weight infants. J Perinatol. 1999;19(4):251-254.

14. Cairns PA, Wilson DC, McClure BG, Halliday HL, McReid M. Percutaneous central venous catheter use in the very low birth weight neonate. Eur J Pediatr. 1995;154(2):145-147.

15. Hoang V, Sills J, Chadler M, Busalani E, Clifton-Koeppel R, Modanlou HD. Percutaneously inserted central catheter for total parenteral nutrition in neonates: complications rates related to upper versus lower extremity insertion. Pediatrics. 2008;121(5):11521159 .

16. Mahieu LM, De Muynck AO, Ieven MM, De Dooy JJ, Goossens HJ, Van Reempts PJ. Risk factors for central vascular catheterassociated bloodstream infections among patients in a neonatal intensive care unit. J Hosp Infect. 2001;48(2):108-116. 